\title{
Laser parameters for efficient biomedical stimulation: A study to increase cognitive response rate
}

\author{
Ji-Sun Kim ${ }^{1}$, Jin-Young Baek ${ }^{1}$, Han-Byeol Oh, Seong-Su Lee and Jae-Hoon Jun* \\ Department of Biomedical Engineering, BK21 Plus Research Institute of Biomedical Engineering, \\ College of Biomedical and Health Science, Konkuk University, Chungju, Korea
}

\begin{abstract}
.
BACKGROUND: The laser is able to irradiate the exact amount of stimulation to an area by a non contact method, and has the advantage of being able to stimulate the local target area.

OBJECTIVE: This study examined an efficient method of laser tactile stimulation using laser parameter combinations.

METHODS: The laser parameters that could cause an increase in the cognitive response rate of human subjects were examined without increasing the amount of total laser energy.

RESULTS: As a result, the appropriate matching parameters such as duty ratio, pulse frequency, and exposure time of laser pulses showed a dominant influence in effectively increasing the tactile response rate of subjects with limited amount of total laser energy.

CONCLUSIONS: This study can be applied to neurophysiology, cognitive research, and clinical laser application.
\end{abstract}

Keywords: Laser tactile stimulation, laser parameter, diode pulse laser, tactile response rate

\section{Introduction}

Development of laser-related equipment is actively underway for diagnosis, treatment, and clinical research. In particular, laser-related research has been actively carried out to relieve or treat diseases using skin and nerve stimulation principles [1-3]. Recently, research on tactile stimulation using lasers has been attempted [4]. Laser tactile stimulation can be applied to cognitive science, which studies active brain areas using EEG, and neurophysiology, which studies the results of nociceptor stimulation $[5,6]$. The laser can be easily applied anywhere on the target area of the skin, and the stimulation device does not directly touch it, so the risk of infection is low and there is an advantage that an accurate amount of stimulation can be exposed. Therefore, the need of clinical laser research in the application on tactile stimulation and cognitive research is increasing compared to chemical stimulation, mechanical stimulation and electrical stimulation [7-11]. Currently, tactile stimulation presentation methods have limitations in precisely controlling parameters such as the intensity of the stimulus, the presenting area

\footnotetext{
${ }^{1}$ These authors contributed equally to this work.

${ }^{*}$ Corresponding author: Jae-Hoon Jun, Department of Biomedical Engineering, College of Biomedical and Health Science, Konkuk University, 268 Chungwondaero, Chungju, Chungbuk 380 701, Korea. Tel.: +82 43 8403799; E-mail: jjun81@ kku.ac.kr.
}

0928-7329/18/\$35.00 (c) 2018 - IOS Press and the authors. All rights reserved

This article is published online with Open Access and distributed under the terms of the Creative Commons Attribution NonCommercial License (CC BY-NC 4.0). 


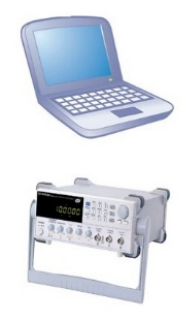

Control System

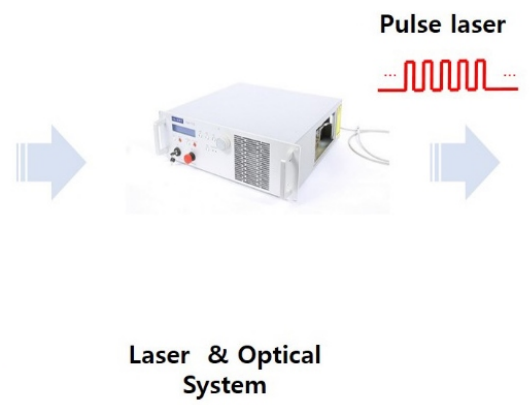

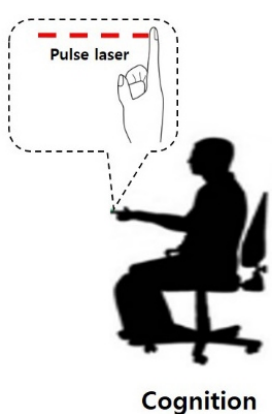

Fig. 1. Schematic of laser stimulation procedure.

and the duration time. For example, electrical stimulation is the most common stimulus to induce pain in animals and humans. However, there is a disadvantage that the size of the system is large, complex, and the intensity and the direction of the stimulus cannot be precisely adjusted. It can also be accompanied by an uncomfortable feeling when presented with the stimulus and when it passes through the skin to stimulate receptors and nerves, excessive stimulation can cause pain. There is an additional disadvantage that an electrode must be attached to the target tissue. Among the mechanical stimulation methods, vibration stimulation has a limitation in showing a small area of touch, and it is difficult to miniaturize the attachment device [12]. Although research on tactile stimulation using lasers capable of precise stimulation is being carried out, only a few studies have examined which parameter is better applied to tactile stimulation by combining various laser parameters in the field of cognitive research. Previously, some studies have been conducted on cognitive response with increasing stimulus intensity [13]. However, there are not many studies regarding efficient cognitive response based on various laser parameter combinations. This study investigated a method of increased stimulation with a small dosage by varying the combination of various laser parameters (pulse energy, frequency, duty ratio, and exposure time) In addition the method of increasing the intensity of the stimulus by increasing the dose of laser was also examined. In this research, a laser tactile stimulus study was carried out using a diode laser. The advantages are that parameters are easily changed while maintaining a stable laser output. The results of this study can be applied to clinical, neurophysiological, and cognitive research. In addition, it can be used valuably in stimulation for cell channel activation, low power laser therapy, optical transfection, etc.

\section{Materials and methods}

\subsection{Laser stimulation procedure}

Figure 1 is a schematic diagram of an experimental setup for laser tactile stimulation. The computer and the function generator were used to precisely control the laser parameters. The laser used in the experiment is a fiber coupled diode laser system (PearlTMTKS-B, nLight Photonics Corp., Santa Clara, CA, USA) with optical fibers connected. The wavelength of this laser is $809 \mathrm{~nm}$ and the pulse width range is variable to $10 \mu \mathrm{s}-\mathrm{CW}$. The experiment was carried out by adjusting the beam diameter to $0.46 \mathrm{~mm}$ using a collimating lens and an optical lens at the tip of the optical fiber where the laser beam is outputted. There were total of 17 subjects (11 Males + 6 Females, Age; 19-25 \pm 1.4 ) and experiments were conducted in a way that the stimulus was presented to the subjects for a certain period of time and the subjects responses were checked. This experiment was approved by the Institutional Review Board at Konkuk University (7001355-201408-HR-032). 
Table 1

Laser parameters used in experiment 1

\begin{tabular}{llccccc}
\hline \multicolumn{2}{c}{ Total energy $=460.05 \mathrm{~mJ}$} & \multicolumn{5}{c}{ Frequency } \\
\cline { 3 - 7 } & & $0.5 \mathrm{~Hz}$ & $5 \mathrm{~Hz}$ & $50 \mathrm{~Hz}$ & $250 \mathrm{~Hz}$ & $500 \mathrm{~Hz}$ \\
\hline Duty ratio 25\% & Exposure time $[\mathrm{s}]$ & 20 & 20 & 20 & 20 & 19.8 \\
& Energy per pulse $[\mathrm{mJ}]$ & 46.05 & 4.605 & 0.4605 & 0.0921 & 0.0465 \\
Duty ratio 50\% & Exposure time $[\mathrm{s}]$ & 10 & 10 & 10 & 10 & 10 \\
& Energy per pulse [mJ] & 92.1 & 9.21 & 0.921 & 0.1842 & 0.0921 \\
Duty ratio 75\% & Exposure time $[\mathrm{s}]$ & 6.67 & 6.67 & 6.67 & 6.67 & 6.67 \\
& Energy per pulse [mJ] & 138.15 & 13.815 & 1.3815 & 0.2763 & 0.13815 \\
\hline
\end{tabular}

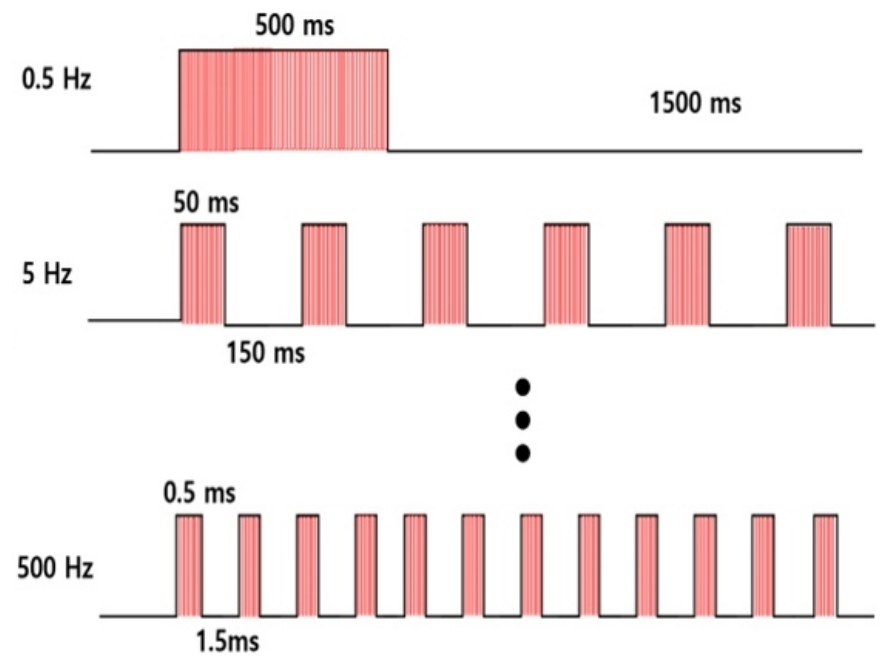

Fig. 2. Stimulus presentation method with fixed total energy.

Two experiments were conducted for the study of laser parameter combinations for tactile stimulation. First, the most effective laser parameters for increasing the response rate of tactile stimulation were studied by changing the energy, exposure time, and frequency after fixing the total energy. The second method was to study effective laser frequency and exposure time according to various combinations of laser parameters by fixing the energy per laser pulse without fixing the total energy.

\subsection{Experiment 1: Laser stimulation with fixed total energy}

Figure 2 shows the laser pulse stimulation method when the duty ratio is $25 \%$. The total energy was fixed at $460.05 \mathrm{~mJ}$, and then the pulse energy, frequency, and exposure time per pulse were changed. The gate timer mode of the diode laser was used for accurate stimulation presentation. In this mode, the exposure time could be precisely controlled by setting the laser pulse pause and pulse duration via the trigger signal connected with the laser. At this time, the peak power of the laser was fixed at $30.7 \mathrm{~W}$ and the energy per pulse was adjusted by changing the pulse width of the laser

Table 1 shows the experimental parameters used in Experiment 1. After fixing the total at $460.05 \mathrm{~mJ}$, experimental parameters such as duty ratio, exposure time, energy per pulse and frequency were selected and combined for various laser parameter combinations. The duty ratios were set to 3 stages, $25 \%, 50 \%$ and $75 \%$, and the frequency was set to $0.5 \mathrm{~Hz}, 5 \mathrm{~Hz}, 50 \mathrm{~Hz}, 250 \mathrm{~Hz}$, and $500 \mathrm{~Hz}$. At this time, the exposure time changes according to the laser pulse width variation and the duty ratio. The exposure 
Table 2

The laser parameters and total energy used for experiment 2

\begin{tabular}{lcccccc}
\hline Exposure time & \multicolumn{5}{c}{ Frequency } \\
\cline { 2 - 7 } & $250 \mathrm{~Hz}$ & $400 \mathrm{~Hz}$ & $550 \mathrm{~Hz}$ & $700 \mathrm{~Hz}$ & $850 \mathrm{~Hz}$ & $1 \mathrm{kHz}$ \\
\hline $5 \mathrm{~s}$ & $1.151 \mathrm{~mJ}$ & $1.842 \mathrm{~mJ}$ & $2.532 \mathrm{~mJ}$ & $3.2235 \mathrm{~mJ}$ & $3.9142 \mathrm{~mJ}$ & $4.605 \mathrm{~mJ}$ \\
& $250 \mathrm{~Hz}$ & $350 \mathrm{~Hz}$ & $450 \mathrm{~Hz}$ & $550 \mathrm{~Hz}$ & $650 \mathrm{~Hz}$ & $750 \mathrm{~Hz}$ \\
$10 \mathrm{~s}$ & $2.3025 \mathrm{~mJ}$ & $3.2235 \mathrm{~mJ}$ & $4.1445 \mathrm{~mJ}$ & $5.0655 \mathrm{~mJ}$ & $5.9865 \mathrm{~mJ}$ & $6.9075 \mathrm{~mJ}$ \\
\hline
\end{tabular}

Table 3

Response rate of subjects with fixed total energy

\begin{tabular}{cccccc}
\hline Duty ratio & $0.5 \mathrm{~Hz}$ & $5 \mathrm{~Hz}$ & $50 \mathrm{~Hz}$ & $250 \mathrm{~Hz}$ & $500 \mathrm{~Hz}$ \\
\hline $25 \%$ & $53 \%$ & $29 \%$ & $35 \%$ & $6 \%$ & $29 \%$ \\
$50 \%$ & $59 \%$ & $41 \%$ & $41 \%$ & $47 \%$ & $35 \%$ \\
$75 \%$ & $76 \%$ & $59 \%$ & $76 \%$ & $76 \%$ & $76 \%$ \\
\hline
\end{tabular}
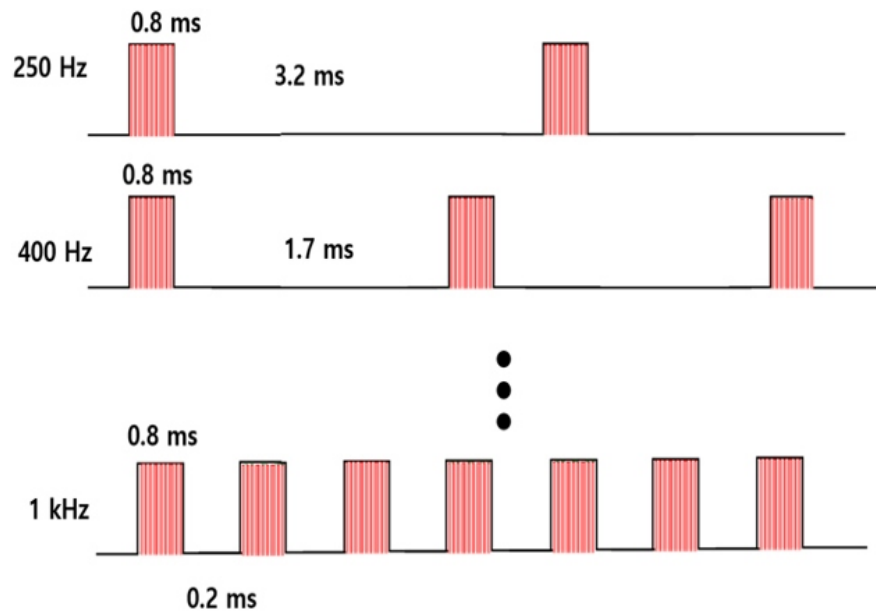

Fig. 3. Stimulus presentation method with fixed pulse width.

time, frequency and the energy per pulse parameters were set based on previous experiments at a point where subjects have almost no pain but still feel contact.

\subsection{Experiment 2: Laser stimulation with fixed pulse width and energy per pulse}

Figure 3 shows the laser stimulation method when energy per pulse is fixed. Experiments were conducted by changing the laser frequency and exposure time after setting the pulse width of laser energy to $0.8 \mathrm{~ms}$. At this time, the total energy according to the stimulus was not fixed. Again, the gate timer mode of the diode laser was used for accurate stimulus presentation and the subjects were asked about the perceived feeling after the end of the stimulation to organize the data.

Table 2 shows the experimental parameters used in Experiment 2. After fixing the laser energy per pulse at $0.7368 \mathrm{~mJ}$, the laser energy exposure time and frequency were combined to obtain cognitive response results. Although the energy per pulse was fixed, it showed that the total energy is changed by the change of frequency and exposure time. For various laser parameter combinations, the frequency was set to $250 \mathrm{~Hz}-1 \mathrm{kHz}$ and the exposure time was set to $5 \mathrm{~s}$ or $10 \mathrm{~s}$. As in Experiment 1, the exposure 


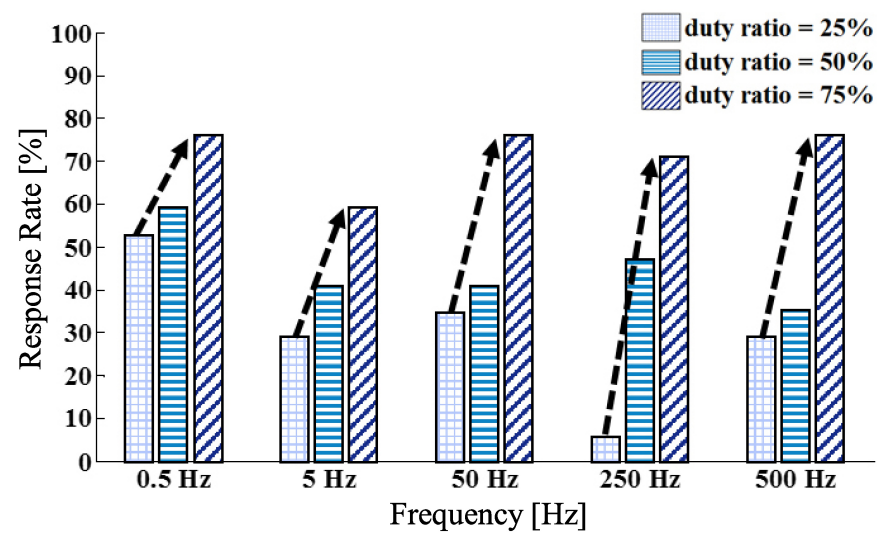

Fig. 4. Cognitive response rate according to laser parameter change with fixed total energy.

time, frequency, and energy per pulse were again set to be the most effective experimental range for the cognitive response through previous experiments.

\section{Results and discussion}

\subsection{Effect of duty ratio on human response}

Table 3 shows the cognitive response rate according to the laser parameter change when the total energy is $460.05 \mathrm{~mJ}$. The response rate in the table is the ratio of the subjects feeling sensation, and the parameters with response rates of $50 \%$ or more is shaded. In the fixed total energy condition, the cognitive response rate was the highest when the frequency was low under the same duty ratio condition. For example, when the duty ratio was $25 \%$, the response rate was $53 \%$ at $0.5 \mathrm{~Hz}$ and $29 \%$ at $500 \mathrm{~Hz}$. This suggests when the duty ratio is fixed, if the frequency is low, the energy per pulse is high. When the duty ratio is $25 \%$, the energy per pulse is $46.05 \mathrm{~mJ}$ at a frequency of $0.5 \mathrm{~Hz}$ and $0.0465 \mathrm{~mJ}$ at $500 \mathrm{~Hz}$. That is, when the same total energy of $460.05 \mathrm{~mJ}$ is irradiated to the human body, the energy per pulse is about 1000 times different according to the duty ratio. This suggests that the cognitive response rate has increased because of it. As a result, even if the same amount of total energy is irradiated, it is possible to obtain a more effective cognitive response rate of the subjects by increasing energy per pulse and decreasing frequency.

Figure 4 shows the trend of cognitive response rate according to changes of frequency and duty ratio. With a frequency of $0.5 \mathrm{~Hz}$, the response rate was $53 \%$ when the duty ratio was $25 \%, 59 \%$ when the duty ratio was $50 \%$, and $76 \%$ when the duty ratio was $75 \%$. This trend was similar in all frequency ranges and the details are illustrated in Figure 4. In addition, under the condition that the total energy was constant, the result of the cognitive response rate according to the frequency change did not show a certain tendency. In other words, it is considered that an effective way to increase the response rate is to increase duty ratio rather than the frequency.

\subsection{Influence of frequency change and exposure time change}

Figure 5 is a graph showing the response rate of the subject perceiving the tactile sensation being exposed to laser stimulation by changing the frequency and exposure time with a fixed pulse width. 


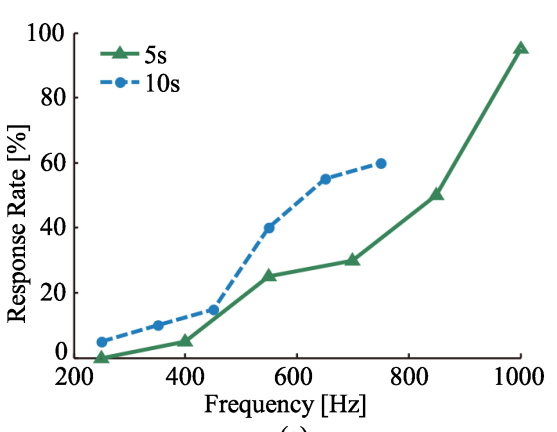

(a)

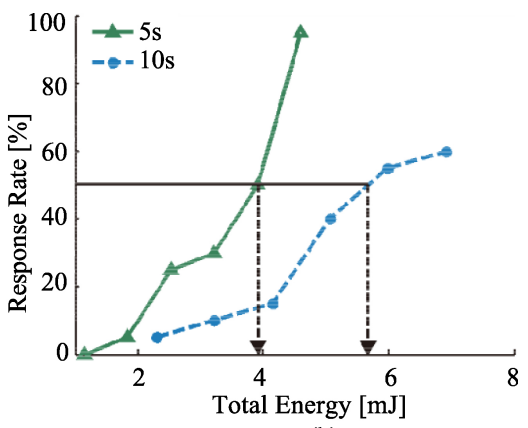

(b)

Fig. 5. Cognitive response rate with laser parameter changes. (a) Change in response rate with increasing frequency, (b) Change in response rate with increasing total energy.

Figure 5a shows the response rate with increasing frequency. The response rate was $25 \%$ at $550 \mathrm{~Hz}$ with an exposure time of $5 \mathrm{~s}$ and $95 \%$ at $1 \mathrm{kHz}$. This figure shows that as the frequency increased, the response rate increased. Even when the exposure time was $10 \mathrm{~s}$, the response rate tended to increase as the frequency increased. This is because, unlike the prior condition with fixed total energy, it is considered that the cognitive response rate is more affected by the frequency when the total energy is not fixed.

Figure $5 \mathrm{~b}$ is a graph showing the response rate of the subjects with total energy increase. Comparing the total energy of the laser exposure time of $5 \mathrm{~s}$ and $10 \mathrm{~s}$ at the response rate of $50 \%$, the total energy is about $3.914 \mathrm{~mJ}$ when the exposure time is $5 \mathrm{~s}$ and $5.507 \mathrm{~mJ}$ when it is $10 \mathrm{~s}$. It can be seen that the efficiency is better at $5 \mathrm{~s}$ where less energy is used showing the same response rate. This indicates that an increase in cognitive response rate can be achieved with small total energy. That is, using a proper combination of exposure time and frequency can induce a higher cognitive response rate with a small amount of total energy.

\section{Conclusion}

This goal of this study was to find the most efficient condition to obtain a tactile response by changing various parameters of a laser. As a result of the experiment it was found that : 1) The higher the duty ratio, the higher the duty response rate than the frequency and exposure time under the same total energy condition, 2) The higher the frequency, the higher the tactile response rate under the same energy per pulse condition. As a main result of the research, it was confirmed that not only the increased exposure amount of the laser but also the proper combination of the frequency and the duty ratio was more effective in increasing the response rate of the subject. The tactile response rate could be increased with a small amount of energy and it was found that biomedical laser stimulation could be achieved effectively though appropriate combination of various laser parameters (duty ratio, frequency, and exposure time). In order to obtain a higher response rate result, a more diverse set of laser parameter combinations is needed in the future and this paper is expected to be applicable to neurophysiology, cognitive research, and clinical research.

\section{Acknowledgments}

This research was supported by Basic Science Research Program through the National Research Foundation of Korea (NRF) funded by the Ministry of Education (No. 2016R1D1A3B03930427) and Basic 
Science Research Program through the National Research Foundation of Korea (NRF) funded by the Ministry of Education (No. 2016R1A6A3A11930264).

\section{Conflict of interest}

None to report.

\section{References}

[1] Hunter J, et al. Effects of low energy laser on wound healing in a porcine model. Lasers in Surgery and Medicine 1984; 3(4): 285-290.

[2] Martins F, et al. Low-level laser therapy modulates musculoskeletal loss in a skin burn model in rats. Acta Cirurgica Brasileira 2015; 30(2): 94-99.

[3] Nussbaum EL, et al. Effects of low intensity laser irradiation during healing of infected skin wounds in the rat. Photonics and Lasers in Medicine 2014; 3(1): 23-36.

[4] Jun J-H, et al. Laser-induced thermoelastic effects can evoke tactile sensations. Scientific Reports 2015; 5: 11016.

[5] Bingel U, et al. Single trial fMRI reveals significant contralateral bias in responses to laser pain within thalamus and somatosensory cortices. Neuroimage 2003; 18(3): 740-748.

[6] Mouraux A, Iannetti GD. Nociceptive laser-evoked brain potentials do not reflect nociceptive-specific neural activity. Journal of Neurophysiology 2009; 101(6): 3258-3269.

[7] Avci P, et al. Low-level laser (light) therapy (LLLT) in skin: Stimulating, healing, restoring. Seminars in Cutaneous Medicine And Surgery. Frontline Medical Communications 2013; 41-52.

[8] De Tommaso M, et al. Update on laser-evoked potential findings in fibromyalgia patients in light of clinical and skin biopsy features. Journal of Neurology 2014; 261(3): 461-472.

[9] Esmaeelinejad M, et al. The effects of low-level laser irradiation on cellular viability and proliferation of human skin fibroblasts cultured in high glucose mediums. Lasers in Medical Science 2014; 29(1): 121-129.

[10] Rossi P, et al. Sympathetic skin response evoked by laser skin stimulation. Functional Neurology 2002; 17(3): 129-132.

[11] Plaghki L, Mouraux A. How do we selectively activate skin nociceptors with a high power infrared laser? Physiology and biophysics of laser stimulation. Neurophysiologie Clinique/Clinical Neurophysiology 2003; 33(6): 269-277.

[12] Moshourab R, et al. Measurement of vibration detection threshold and tactile spatial acuity in human subjects. Journal of Visualized Experiments: JoVE 2016; 115.

[13] Kim J-S, et al. Study of somesthesis according to change in pulse diode laser parameters. Bio-Medical Materials and Engineering 2015; 26(s1): S1001-S1008. 